\title{
Concept transfer as a function of age and variability of irrelevant features during acquisition
}

\author{
LORRAINE A. LOW, ELLEN COSTE, and CYNTHIA KIRKUP \\ Framingham State College, Framingham, Massachusetts 01701
}

\begin{abstract}
The influence of two different levels of variability of irrelevant information during acquisition on concept transfer for second-, fourth-, and sixth-grade children was investigated. Two different figures, faces, or houses constituted an additional between-subjects variable. The shapes of specific features for faces (e.g., eyes, ears, etc.) and for houses (e.g., doors, windows, etc.) constituted the irrelevant dimensions varied. One feature for each figure constituted the relevant dimension. One or three irrelevant features resulted in two levels of irrelevant dimension variability. The number of new irrelevant dimensions $(T)$ that varied during transfer constituted a within-subjects variable. The results differed for the two figures but generally indicated a decrement in errors over all levels of $\mathrm{T}$, with increase in variability during acquisition for older but not for younger children.
\end{abstract}

The influence of variability during acquisition on concept transfer as a function of age was examined in a study by Low, Woolverton, and Lusignan (1976). In that study, schematic faces were presented to firstand fourth-grade children. Dimensions were defined by the particular facial features; the attributes were defined by the shapes of these features. The shape of the ear was relevant to category placement. During acquisition, the shape of the moustache varied orthogonally with the ears (idiosyncratic stimuli) for one group (MV) and was held constant (common stimuli) for the second group (MC). In transfer, the faces contained either an old moustache (presented during acquisition) or a new one (never presented previously), both old ears, and a new one. The measure of interest was the number of errors in which the old ears were identified as new. It was found that the first graders made a greater number of errors in the MV group (irrelevance was common). These differences were significantly reversed for the fourth graders.

The previous findings have broad implications for developmental differences in category abstraction. Age differences in processing irrelevant information appear to be critical variables associated with age-related improvement in category abstraction. The present study was therefore designed to extend these findings. It tested the ability of older children to generalize information about irrelevance from idiosyncratic stimuli during

The authors wish to thank the administrators, teachers, and students of the following Massachusetts elementary schools for their participation: Miller School (Holliston), Saint Bridget's School (Framingham), Brown School (Wellesley), and Schofield School (Wellesley). Requests for reprints should be sent to Lorraine A. Low, Psychology Department, Framingham State College, Framingham, Massachusetts 01701. acquisition to different idiosyncratic stimuli in transfer. In this study, those irrelevant dimensions or features that varied (idiosyncratically) during acquisition, remained constant (common) during transfer. Similarly, those irrelevant dimensions that were constant during acquisition constituted the population from which variable dimensions were chosen during transfer. Variability in the present study was defined by the number of bilevel varying dimensions, rather than by the presence or absence of variation, as in the previous study. An additional variable, the degree of transformation (T) was defined by the number of unchanging irrelevant dimensions during acquisition that were subsequently varied during transfer. The errors in this study were defined by the number of times one of the previously presented categories was called "neither" (neither one of the old categories) during transfer. It is expected that (1) increase in number of variable dimensions during acquisition will decrease errors during transfer for older children, (2) errors will increase with increase in variability for younger children, and (3) the above differences will be a positive function of $\mathrm{T}$.

\section{METHOD}

\section{Subjects}

Subjects were second-, fourth-, and sixth-grade children, 32 from each grade, randomly selected from local elementary schools. They were randomly and equally assigned to one of four conditions, two variability conditions, and two conditions with specific figures (SF).

\section{Stimulus Material and Design}

The stimuli consisted of figures of faces or houses cut from red construction paper and pasted on posterboard. The two different figures constituted the SF condition. There were 10 
different features for each figure. Examples of some features were eyes, ears, and noses for faces and roofs, doors, and porches for houses. The two shapes of the ears for faces and the porches for houses were relevant to category assignment. Irrelevant variation was defined by two shapes of each feature or dimension. For one variation condition $\left(V_{1}\right)$, the shape of the irrelevant feature varied orthogonally with the relevant feature; for the other $\left(V_{3}\right)$, the shapes of three irrelevant features varied orthogonally with each other and with the relevant feature. These features remained constant during transfer, using one of the two acquisition shapes. The irrelevant features varied during transfer were chosen from the population of features that remained constant during acquisition. The number of irrelevant features that varied was une, three, or five, defined by the level of $T$. These levels were $T_{1}, T_{3}$, and $T_{5}$, respectively. When any feature varied during transfer, it contained two new shapes. Half of the transfer stimuli contained a new relevant shape; half of the remaining stimuli contained one of the old relevant shapes, and the other half contained the other old relevant shape. The old and new relevant shapes constituted a transfer variable $(O, N)$. The new relevant shape was introduced so that the children could respond correctly with the term "neither." However, the primary interest was in the number of old relevant categories incorrectly responded to as "neither."

The design was a 3 (age) by 2 (variability) by 2 (SF) by 3 (T) by $2(\mathrm{O}, \mathrm{N})$ mixed factorial, with the last two factors as withinsubjects variables.

\section{Procedure}

The stimuli for the variability conditions were assigned to trials with the restriction that every stimulus occur once within each block of trials. The number of different stimuli in each block were 4 and 16 for the $V_{1}$ and $V_{3}$ conditions, respectively. Fach child was tested individually. He was told that this was a game in which each picture was to be placed in one of two categories. For faces, the child was told that the categories were to be labeled "Joe" or "Eddie," and for houses, "Joe's house" or "Eddie's house." All possible features were pointed out, and the child was informed that the categories would be determined by the different shapes of only one of the features. The task was to label the categories correctly, and the subject was informed after each response whether it was correct or incorrect. Every child was run to a criterion and given a minimum of 16 and a maximum of 32 trials. Those children who did not reach the criterion of six correct on eight consecutive trials were eliminated from the study.

During transfer, the three levels of $T$ and two levels of $O, N$ were orthogonally combined and randomly assigned to each block of six trials. The particular combination of irrelevant shapes of features was chosen randomly from the total population of possibilities for each level of T. Any combination was used only once. The child was told before transfer began that pictures would once again be presented to him. He was informed that some of the pictures belonged to the Joe or Eddie category, but that others did not belong to either. If the stimuli did not belong to either category, the subject was to call it "neither." The child was not told whether his response was correct. A total of 36 trials was presented in transfer.

\section{RESULTS}

\section{Acquisition}

The response score was the total number of errors in identifying categories. The number of trials to criterion was not analyzed, since there was a narrow range in scores on this measure. An analysis of variance was performed on the 3 (age) by 2 (variability) by 2 (SF) between-subjects factorial design. None of the main effects or interactions was statistically significant.

\section{Transfer}

The response measure was the total number of errors in which either old category was identified as "neither." An analysis of variance was performed on the 3 (age) by 2 (variability) by 2 (SF) by 2 (T) mixed factorial design, in which $\mathrm{T}$ was the only within-subjects variable. The mean number of errors as a function of the major variables of variability, $\mathrm{T}$, and $\mathrm{SF}$ are presented in Figure 1. Age effects were statistically significant $[F(2,84)=7.33, p<.01]$. Means of 7.08, 6.60, and 3.00 for second-, fourth-, and sixth-grade children, respectively, indicated that errors decreased with age. Tukey's test for specific comparisons yielded a nonstatistical difference between the number of errors for the second and fourth graders, but a statistically significant difference $(p<.01)$ between the second and sixth graders and between the fourth and sixth graders. The variability condition approached but did not reach statistical significance $[F(1,84)=3.38, .10>p>.05]$, indicating a trend toward fewer errors with increased variability. The SF effects were statistically significant $[F(1,84)=4.41, p<.05]$, with faces resulting in more errors than houses (means of 6.57 and 4.56 for faces and houses, respectively). The effects of $\mathrm{T}$ were statistically reliable $[F(2,168)=60.77, p<.01]$, indicating a linear increment in number of errors with increase in $\mathrm{T}$ (see Figure 1).

The interaction of Age by $T$ was statistically significant $[F(4,168)=7.22, p<.01]$. It is evident from inspection of Figure 1 that the rate of increment in errors as a function of $\mathrm{T}$ decreased with increase in age. The Variability by SF by $\mathrm{T}$ interaction was statistically significant $[F(2,168)=9.39, p<.01]$. For faces, the difference between errors for $V_{1}$ and $V_{3}$ decreased over $\mathrm{T}$, but for houses, this difference increased over levels of T. Neither the Age by Variability nor the Age by Variability by $T$ interaction was statistically significant ( $F<1$ in both instances). The Age by Variability

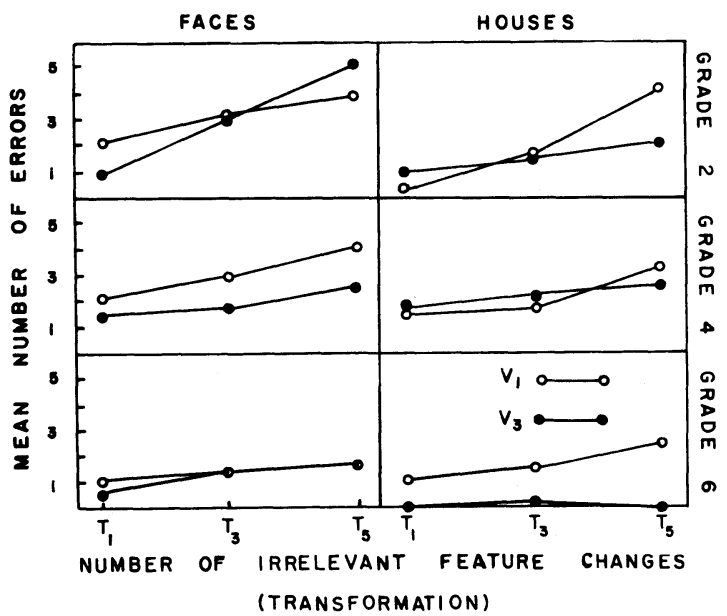

Figure 1. Mean number of errors in calling the "old" categories "neither" as a function of grade level (age), acquisition variability $\left(V_{1}, V_{3}\right)$, and degree of transformation during transfer $(T)$ for houses and faces. 
by $\mathrm{SF}$ by $\mathrm{T}$ interaction was statistically significant $[F(4,168)=3.41, p<.05]$. It is evident from Figure 1 that the Age by Variability by $\mathrm{T}$ interaction was different for faces and houses. Separate analyses of variance were therefore performed for faces and houses. For faces, this interaction approached statistical significance $[F(4,84)=2.33, .10>p>.05]$. This trend suggests that the $V_{3}$ condition, compared with the $V_{1}$ condition, showed no consistent effect over levels of $\mathrm{T}$ for the second graders but resulted in consistently fewer errors over all levels of $T$ for the fourth graders. There were no differences for the sixth graders. For houses, the Variability by $\mathrm{T}$ interaction was statistically significant $[F(4,84)=2.86, p<.05]$. It is evident from Figure 1 that the effects of variability in reducing the error score were evident only at $T_{5}$ for second and fourth graders but were consistent for all levels of $\mathrm{T}$ for the sixth graders.

\section{DISCUSSION}

The findings of the present study indicate that, although children learn what is relevant to categorization, they do not necessarily discriminate the components that are irrelevant to the solution of the problem. In the present study, the increase in errors with $\mathrm{T}$ points to the importance of common stimuli in maintaining the concept. With change in these components as defined by an increase in $\mathrm{T}$, the number of errors dramatically increased. The Age by $\mathrm{T}$ interaction also points to the decrease in this tendency to include irrelevant components with age.

The major hypothesis of this study was that older children would benefit from increase in number of idiosyncratic stimuli as defined by increased variability in discriminating the relevant from the irrelevant components of the stimulus complex. Younger children, on the other hand, would be influenced by the salience of variability and therefore would demonstrate poorer transfer with an increase in the number of variable stimuli. Thus it was expected that the number of errors in identifyirig the old stimuli as "neither" would decrease with variability of irrelevant stimuli for older children and increase for younger children. The hypothesis was only partially supported by the data. The figures used proved to be a variable that interacted significantly with the other variables under investigation. Indirect support for the hypothesis came from the findings that the consistency of the positive effects of variability over levels of $T$ was related to age. With houses, increase from $V_{1}$ to $V_{3}$ resulted in fewer errors for the sixth graders across all levels of $\mathrm{T}$. This consistency was not present for either the second or the fourth grade children. With faces, the fourth graders as contrasted with the second graders showed the positive effects of increased variability over all levels of $\mathrm{T}$. However, for the sixth graders, there were no differences between $V_{1}$ and $V_{3}$, indicating, perhaps, that with faces there was a ceiling effect.

The SF effect could have obscured any differences associated with variability and with the Variability by Age interaction. The differential responding to faces and houses might have reflected different preexperimental habits to the two concepts.

The present findings suggest, however, that variability of irrelevant information is age related, and that the effects of specific figures used might have obscured the predicted effects.

\section{REFERENCE}

Low, L. A., Woolverton, M., \& Lusignan, R. Influence of variability of irrelevant features on concept transfer in children. Bulletin of the Psychonomic Society, 1976, 8, 422-424.

(Received for publication August 7, 1978.) 